\title{
Myeloid cell leukemia-1 regulates the cell growth and predicts prognosis in gastric cancer
}

\author{
WAN-SIK LEE, YOUNG-LAN PARK, NURI KIM, HYUNG-HOON OH, DONG-JUN SON, \\ MI-YOUNG KIM, CHAN-YOUNG OAK, CHO-YUN CHUNG, HYUNG-CHUL PARK, \\ JONG-SUN KIM, DAE-SEONG MYUNG, SUNG-BUM CHO and YOUNG-EUN JOO \\ Department of Internal Medicine, Chonnam National University Medical School, \\ Dong-ku, Gwangju 501-757, Republic of Korea
}

Received December 9, 2014; Accepted January 29, 2015

DOI: 10.3892/ijo.2015.2890

\begin{abstract}
The expression of myeloid cell leukemia-1 (Mcl-1), a member of the anti-apoptotic Bcl-2 protein family, has been associated with tumor progression and adverse patient outcome. The aims of current study were to evaluate whether Mcl-1 affects the survival or death of gastric cancer cells, and to investigate the prognostic value of its expression in gastric cancer. PcDNA3.1-Mcl-1 expression and Mcl-1 siRNA vectors were used to overexpress and silence Mcl-1 expression in gastric cancer cell lines including SNU638 and TMK1, respectively. Immunohistochemistry was used to determine the expression of Mcl-1 in gastric cancer tissues. Apoptosis was determined by the TUNEL assay, and cell proliferation was determined by immunostaining with a Ki-67 antibody. Mcl-1 knockdown induced apoptosis through the upregulation of caspase-3, and -7, and PARP activity, and the release of Smac/DIABLO and Omi/HtrA2 into the cytoplasm. Additionally, cell cycle arrest occurred due to decrease of cyclin D1, cell division cycle gene $2(\operatorname{cdc} 2)$, and cyclin-dependent kinase 4 and 6 . In contrast, overexpression of Mcl-1 inhibited apoptosis and cell cycle arrest. Mcl-1 knockdown did not suppress tumor cell proliferation in gastric cancer cells, whereas overexpression of Mcl-1 enhanced tumor cell proliferation. The JAK2 and STAT3 signaling cascades were significantly blocked by Mcl-1 knockdown. The mean Ki-67 labeling index (KI) value of Mcl-1 positive tumors was significantly lower than that of Mcl-1 negative tumors. However, there was no significant difference between Mcl-1 expression and the apoptotic index (AI). Mcl-1 expression was significantly increased in gastric cancer tissues compared to normal gastric mucosa tissues, and was associated with age, tumor size, stage, depth of invasion,
\end{abstract}

Correspondence to: Professor Young-Eun Joo, Department of Internal Medicine, Chonnam National University Medical School, 8 Hak-Dong, Dong-ku, Gwangju 501-757, Republic of Korea

E-mail: yejoo@chonnam.ac.kr

Key words: myeloid cell leukemia-1, apoptosis, proliferation, prognosis, stomach neoplasm lymph node metastasis and poor survival. Our study showed that Mcl-1 regulates the cell growth and might be a potential prognostic marker for gastric cancer.

\section{Introduction}

Although the incidence rate for gastric cancer has steadily declined in past decades, gastric cancer is the second leading cause of cancer-related deaths, with one of the highest mortality rates in the world $(1,2)$. Therefore, identifying the molecular changes that occur in gastric carcinogenesis would contribute to an improved understanding of the pathogenesis of this disease, and would offer the potential for subsequent advances in prevention, earlier diagnosis and perhaps better intervention strategies.

Cancer progression, such as tumor invasion and metastasis results from the accumulation of alterations in genes involved in cell proliferation, mitogenesis, invasiveness and angiogenesis as well as in the inhibition of apoptosis (3-5). In particular, it has been widely accepted that tumor cell proliferation and inhibition of apoptosis are the most crucial steps in tumor invasion and metastasis $(6,7)$.

The Bcl-2 (B-cell lymphoma-2) family of proteins, which consists of anti-apoptotic and pro-apoptotic members, is a critical regulator for the mitochondrial pathway of apoptosis through controlling the integrity of the outer mitochondrial membrane (8). Bcl-2 family members can be divided into three groups, based on their conserved homology domains, named the Bcl-2 homology $(\mathrm{BH})$ domains. The first group is anti-apoptotic proteins, which have all four $\mathrm{BH}$ domains (BH1-BH4) including Bcl-2, Bcl-xL, myeloid cell leukemia-1 (Mcl-1), Bcl-w and A1. The second group is pro-apoptotic proteins, which contain three conserved domains (BH1-BH3) including Bax, Bak and Bok. The third group is the BH3-only pro-apoptotic proteins including Bid, Bim, Puma, Noxa, Bad, Bmf, Hrk and Bik (8).

Anti-apoptotic Bcl-2 family members promote cell survival by inhibiting pro-apoptotic proteins, directly binding and blocking activation of caspases by cytochrome $\mathrm{C}$ and preserving the integrity of the mitochondrial outer membrane against apoptotic stimuli. Anti-apoptotic Bcl-2 family members are known to be expressed at high levels in variable 
human cancers, and allow cells to evade apoptotic signals and attain a neoplastic state (9-11).

Mcl-1 is a potent anti-apoptotic Bcl-2 protein and exerts its anti-apoptotic function by heterodimerizing with other Bcl-2 family members and preventing the permeabilization of the mitochondrial outer membrane (12). It is overexpressed in many human cancers and can confer resistance to apoptotic signaling and treatment $(13,14)$. In addition, the expression of Mcl-1 is associated with tumor progression and adverse patient outcome in many human cancers including gastric cancer (15-21). Recently, several molecules that specifically inhibit Mcl-1 have been discovered, providing a potential role for Mcl-1 as a therapeutic target in cancer (22-25).

The aims of current study were to evaluate whether Mcl-1 affects the survival or death of gastric cancer cells, and to investigate the prognostic value of its expression in gastric cancer.

\section{Materials and methods}

Patients and tissue samples. For this study, formalin-fixed, paraffin-embedded tissue blocks were obtained from 139 randomly chosen patients who had undergone surgery for gastric cancer at the Chonnam National University Hwasun Hospital (Jeonnam, Korea) between January 1997 and December 1998. Patients who had received preoperative chemotherapy or irradiation before surgery were excluded from this study. The clinicopathologic parameters at the time of surgery were reviewed through the medical records. Tumor staging was conducted in accordance with the American Joint Committee on Cancer (AJCC) staging system (26). Survival was measured from the time of surgery until follow-up on December 31, 2012. This study was approved by the Institutional Review Board of Chonnam National University Hwasun Hospital.

Cell culture and siRNA transfection. Human gastric cancer cell lines, SNU638 and TMK1 were obtained from the American Type Culture Collection Line Inc. Cell lines were cultured in RPMI-1640 (Hyclon, Loan, UT, USA) supplemented with 10\% fetal bovine serum and antibiotics. Mcl-1 and scramble siRNA were purchased from Bioneer (Daejeon, Korea) and Qiagen (Germantown, MD, USA), respectively. Mcl-1 construction was subcloned into a pcDNA3.1 vector (Invitrogen, Carlsbad, CA, USA). The transfection of the specific gene was performed with Lipofectamine ${ }^{\mathrm{TM}}$ RNAiMAX (Invitrogen) and Fugene 6 (Promega, Madison, WI, USA) according to the manufacturer's recommendations, respectively. In addition, gene-transfected cells were selectively treated with pharmacological caspase inhibitor, Z-VAD-FMK (5 $\mu \mathrm{M} / \mathrm{ml}, \mathrm{MBL}$, Woburn, MA, USA) and 5-Fluorouracil (5-FU) $(10 \mu \mathrm{g} / \mathrm{ml}$, Choong-Wae, Chung-Nam, Korea).

Western blotting. Total cell proteins were prepared with RIPA ${ }^{\circledR}$ reagent (Thermo Scientific, Rockford, IL, USA). Cytosolic and mitochondrial proteins were isolated with the Mitochondria Isolation kit (Thermo Scientific) according to the manufacturer's recommendations. The protein was subjected to SDS-polyacrylamide electrophoresis and electrotransferred onto a PVDF membrane. Blots were visualized by the luminescent image analyzer LAS-4000 (Fujifilm, Tokyo, Japan) with an enhanced chemiluminescence detection system HRP substrate. The following antibodies were used; antibodies against Mcl-1, cleaved caspase-3, -7, cleaved poly (ADP-ribose) polymerase (PARP), second mitochondria-derived activator of caspases/direct inhibitors of apoptosis protein (IAP) binding protein with low PI (Smac/DIABLO), Omi/high-temperature requirement protein $\mathrm{A} 2$ (Omi/HtrA2), CoxIV, cyclin D1, cyclin D3, phospho-cell division cycle gene 2 (phospho-cdc2), cyclin-dependent kinase 4 (CDK4), CDK6, p21, p27, phosphoextracellular signal-regulated kinase1/2 (phospho-ERK1/2), phospho-glycogen synthase kinase-3 $\beta$ (phopho-GSK3 $\beta$ ), phospho-AKT, Janus kinase 2 (JAK2), phospho-JAK2, Signal transducers and activators of transcription-3 (STAT3), and phopho-STAT3. They were purchased from Cell Signaling Technology (Danvers, MA, USA). Antibodies against $\beta$-tubulin and GAPDH were used and purchased from Santa Cruz Biotechnology (Santa Cruz, CA, USA).

Cell proliferation assay. Proliferation of transfected cells was measured with the EZ-CyTox Cell Viability Assay kit (Daeil Lab Inc., Seoul, Korea), which contains WST-1. EZ-CyTox cell viability reagent was applied at $37^{\circ} \mathrm{C}$ and allowed to develop. Cell viability was measured at $490 \mathrm{~nm}$ absorbance using a microplate reader (Infinite M200, Tecan, Austria GmbH, Austria). All experiments were conducted at least in triplicate.

Flow cytometric analysis. For the apoptosis assay, attached cells were collected and stained in APC Annexin V and 7-amino-actinomycin D (7-AAD) (BD Biosciences, San Diego, CA, USA). For cell cycle analysis, cells were incubated in ribonuclease A (Sigma-Aldrich, St. Louis, MO, USA) and propidium iodide (PI) (Sigma-Aldrich). The apoptotic cells and cell cycle phase were analyzed on the BD Cell Quest ${ }^{\circledR}$ version 3.3 (Becton Dickinson, San Jose, CA, USA) and WinMDI version 2.9 (The Scripps Research Institute, San Diego, CA, USA), respectively.

Immunohistochemical analysis. Immunostaining was performed using a Dako Real ${ }^{\mathrm{TM}}$ Envision HRP/DAB detection system (Dako Cytomation, Glostrup, Denmark). After antigen retrieval, the endogenous peroxidase activity was blocked with a peroxidase-blocking solution and incubated with polyclonal rabbit anti-human Mcl-1, Ki-67 (Dakopatts, Glostrup, Denmark) in primary diluent solution (Invitrogen) overnight at $4^{\circ} \mathrm{C}$. After rinsing in TBST, tissues were stained using 3,3-diaminobenzidine (DAB) liquid, counterstained with Mayer hematoxylin (Sigma-Aldrich). Stained tissues were viewed and photographed using a light microscope (Olympus, Tokyo, Japan).

Evaluation of Mcl-1 expression. Immunoreactivity of Mcl-1 expression was evaluated independently on the basis of the intensity and extent of staining by two observers without knowledge of the clinicopathological data. The staining intensity was graded on a scale of four: 0 , no staining of cancer cells; 1 , weak staining; 2 , moderate staining; 3 , strong staining. The staining extent was also graded on a scale of four: 0 , none; $1,<10 \% ; 2,10-50 \% ; 3,>50 \%$. The intensity rating was multiplied by the extent rating to obtain a mean overall score. The mean overall score of 4.0 was chosen as the cut-off point for the positive status of Mcl-1 expression. 
A

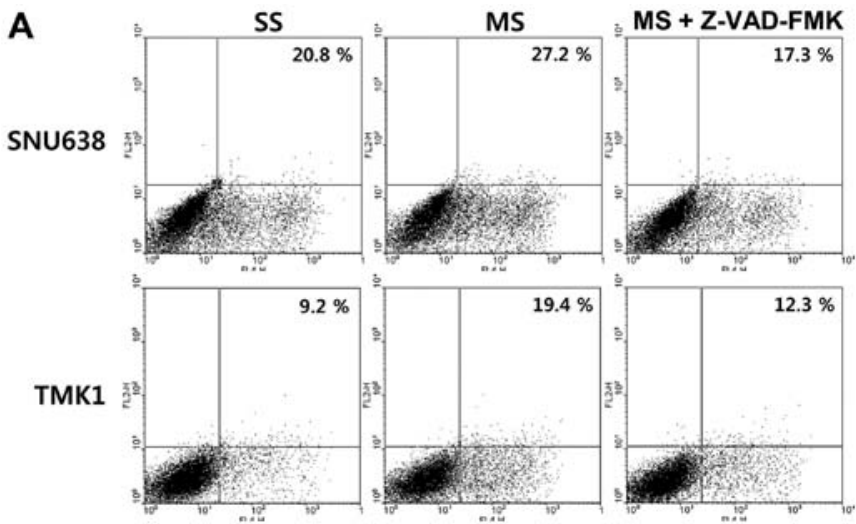

B
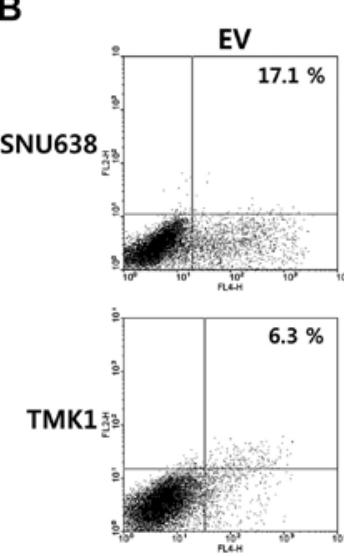

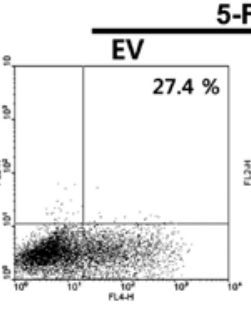

5-FU
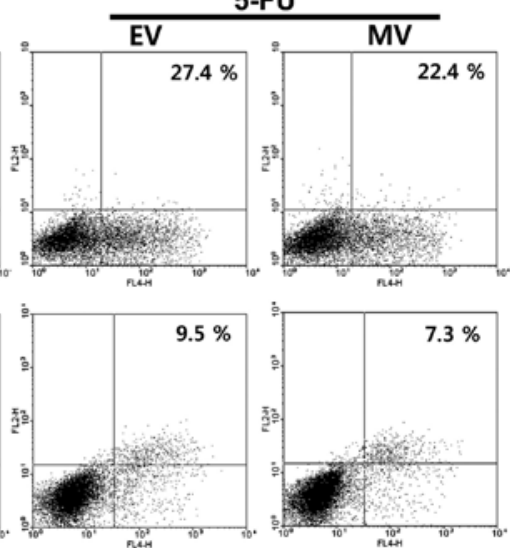

C

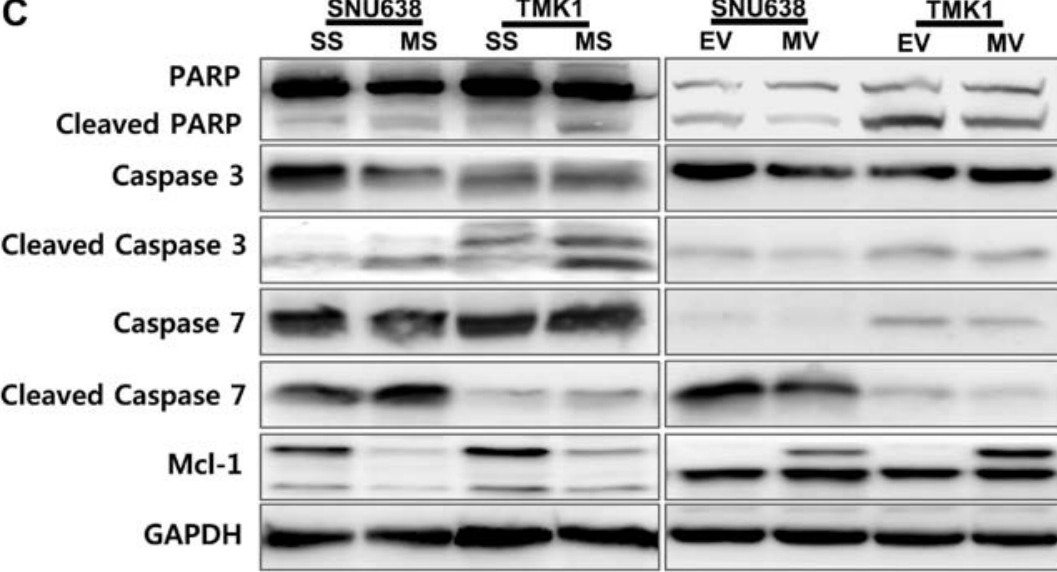

D
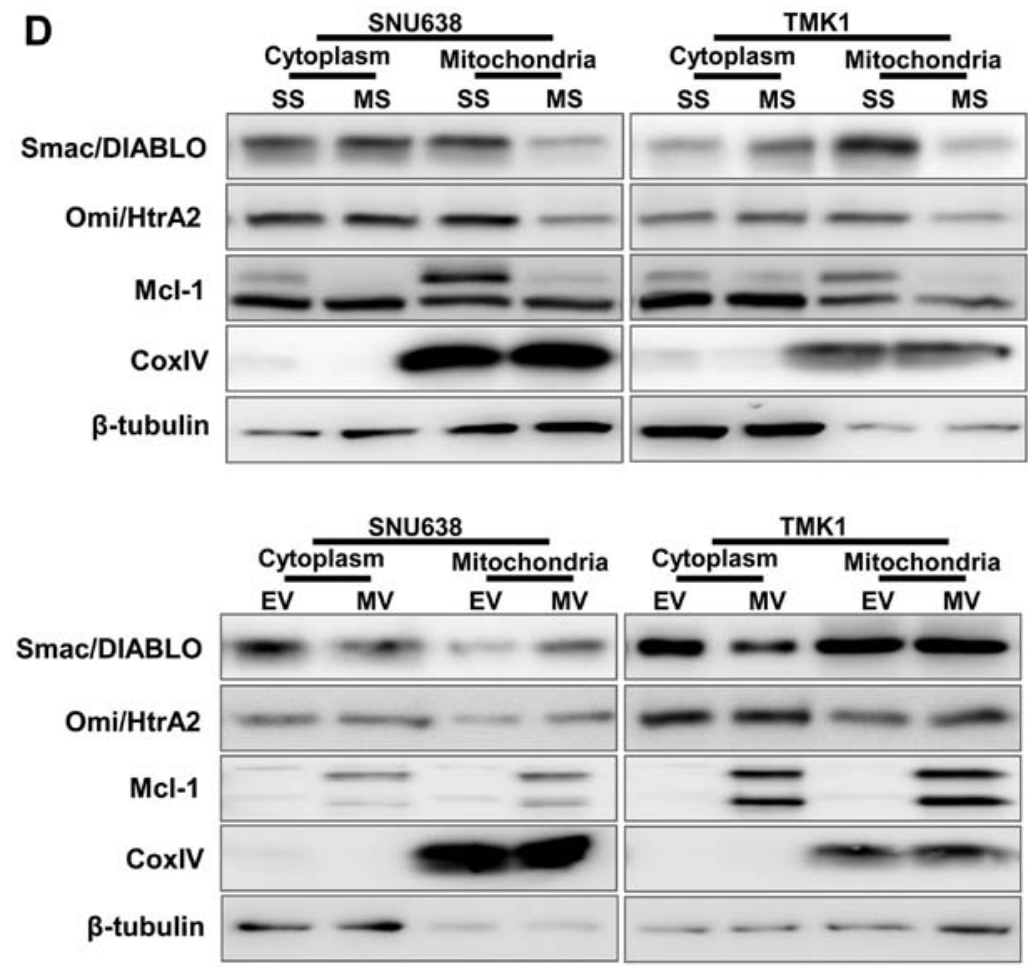

Figure 1. The impact of Mcl-1 on apoptosis in gastric cancer cells. (A) The cell apoptotic rate induced by the transfection of Mcl-1 siRNA significantly increased, compared with that induced by the transfection of the scramble siRNA (20.8 and 9.2 vs. 27.2 and 19.4\%) in the SNU638 and TMK1 cells, and the increases were blocked by treatment with a pharmacological caspase inhibitor, Z-VAD-FMK. (B) Overexpression of Mcl-1 by transfection of pcDNA3.1-Mcl-1 inhibited the apoptosis of SNU638 and TMK1 cells in response to 5-FU. (C) The cleaved caspase-3, -7, and PARP expression levels were upregulated in the SNU638 and TMK1 cells after Mcl-1 knockdown and downregulated after the overexpression of Mcl-1. (D) The release of Smac/DIABLO and Omi/HtrA2 into the cytoplasm was induced by Mcl-1 knockdown and inhibited by the overexpression of Mcl-1. PARP, poly (ADP-ribose) polymerase; Smac/DIABLO, second mitochondria-derived activator of caspases/direct IAP binding protein with low pI; Omi/HtrA2, Omi/high-temperature requirement protein A2; SS, scramble siRNA; MS, Mcl-1 siRNA; EV, empty-pcDNA3.1; MV, pcDNA3.1-Mcl-1; 5-FU, 5-fluorouracil. 
A
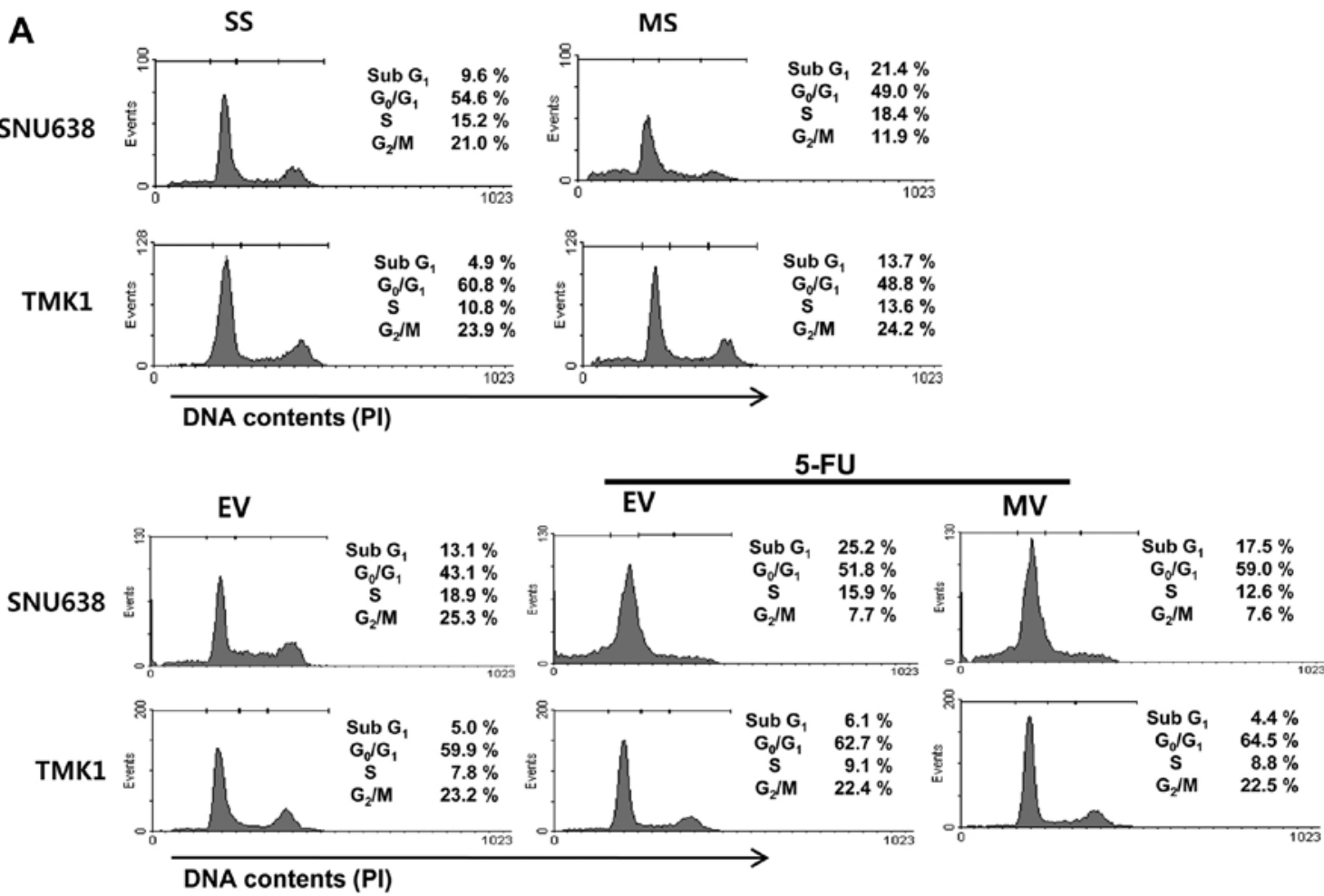

DNA contents (PI)

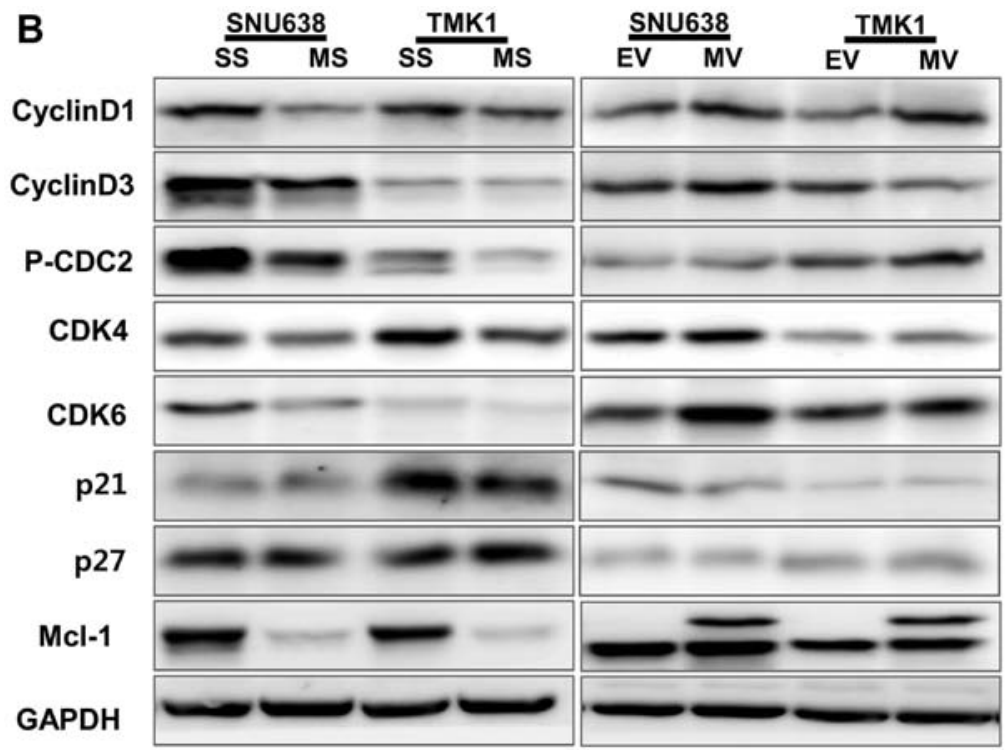

Figure 2. The impact of Mcl-1 on cell cycle distribution in gastric cancer cells. (A) Mcl-1 knockdown resulted in cell cycle arrest in the sub G1 phase of SNU638 and TMK1 cells. The cell cycle arrest in the sub-G1 phase induced by 5-FU treatment was inhibited by the overexpression of Mcl-1. (B) The cyclin D1, P-cdc2, CDK4 and CDK6 protein levels were significantly decreased by Mcl-1 knockdown, and increased by the overexpression of Mcl-1 in SNU638 and TMK1 cells. However, p21 and p27 protein levels remained unchanged by knockdown and overexpression of Mcl-1. SS, scramble siRNA; MS, Mcl-1 siRNA; EV, empty-pcDNA3.1; MV, pcDNA3.1-Mcl-1; 5-FU, 5-fluorouracil; cdc2, cell division cycle gene 2; CDK, cyclin-dependent kinase.

Assessment of tumor cell proliferation and apoptosis. The proliferative ability of tumor cells was presented with the Ki-67 labeling index (KI). The KI was determined by the number of Ki-67-positive nuclei per 1000 tumor cell nuclei. For the detection and quantification of apoptosis, the DeadEnd $^{\mathrm{TM}}$ Colorimetric terminal deoxynucleotidyl transferase dUTP nick end labeling (TUNEL) system (Promega) was used, following the instructions of the manufacturer. TUNEL-positive, darkly stained nuclei or nuclear fragments with a cytoplasmic halo were considered as positive apoptotic cells. The apoptotic index (AI) was expressed as a number of TUNEL positive nuclei including apoptotic body among 1000 tumor cell nuclei.

Statistical analysis. Statistical analysis was performed with the Statistical Package for the Social Sciences (SPSS/PC+ 15.0, Chicago, IL, USA). The expression of Mcl-1 with relation to various clinicopathological parameters was assessed with the $\chi^{2}$ test and Fisher's exact test. The survival rates of patients was estimated with the Kaplan-Meier method and analyzed using a log-rank test. The relationship between Mcl-1 expression and KI or AI was evaluated by the Student's t-test. In the 

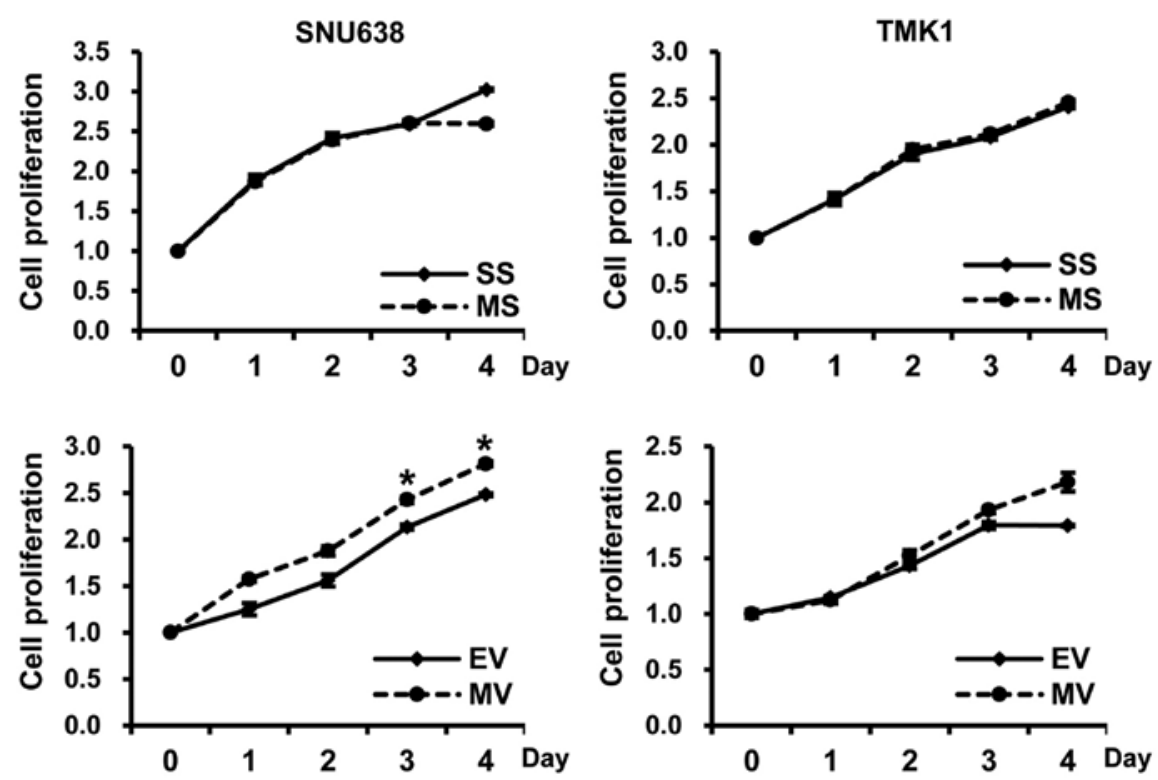

Figure 3. The impact of Mcl-1 on the proliferation of gastric cancer cells. The proliferating cells, as determined by absorbance, were significantly increased in the pcDNA3.1-Mcl-1-transfected SNU638 cells compared to the empty-pcDNA3.1-transfected cells at 3 and 4 days (mean \pm SE, $n=3$; ${ }^{*} \mathrm{p}<0.05$ ), but there was no significant difference in TMK1 cells. In addition, in all tested cells, there was no significant difference regarding cell proliferation between Mcl-1 siRNA and scramble siRNA transfected cells. SS, scramble siRNA; MS, Mcl-1 siRNA; EV, empty-pcDNA3.1; MV, pcDNA3.1-Mcl-1.

in vitro experiments, statistical significance was determined by a Student's t-test. Differences were considered significant when $\mathrm{p} \leq 0.05$.

\section{Results}

Impact of Mcl-1 on apoptosis in gastric cancer cells. To explore the effect of Mcl-1 on the survival or death of gastric cancer cells, Mcl-1 siRNA or pcDNA3.1-Mcl-1 were used to silence or overexpress Mcl-1 expression in gastric cancer cell lines, SNU638 and TMK1, respectively. Mcl-1 expression in all tested cells, showed a specific decrease by transfection with Mcl-1 siRNA and a specific increase by transfection with pcDNA3.1-Mcl-1 (data not shown). To study the effect of Mcl-1 on apoptosis, we performed flow cytometric analyses. The cell apoptotic rate induced by transfection of Mcl-1 siRNA significantly increased, compared with that induced by transfection of the scramble siRNA (20.8 and 9.2 vs. 27.2 and $19.4 \%$ ) in the SNU638 and TMK1 cells, and these increases were blocked by treatment with the pharmacological caspase inhibitor Z-VAD-FMK (Fig. 1A). 5-FU is well known to induce apoptosis and affect the cell cycle of cancer cells. Overexpression of Mcl-1 by transfection of pcDNA3.1-Mcl-1 inhibited the apoptosis of the SNU638 and TMK1 cells in response to 5-FU (Fig. 1B).

To determine the activation of caspases during knockdown and overexpression of Mcl-1, we further investigated caspase-specific activities. The cleaved caspase-3, -7, and PARP expression levels were upregulated in the SNU638 and TMK1 cells after Mcl-1 knockdown and downregulated after overexpression of Mcl-1 (Fig. 1C). The release of Smac/ DIABLO and HtrA2/Omi from the mitochondrial membrane to the cytoplasm triggers activation of the caspase cascade and ultimately induces apoptosis. To analyze the release of Smac/ DIABLO and Omi/HtrA2 to the cytoplasm due to Mcl-1 expression, we examined the expression of SmacC/DIABLO and $\mathrm{Omi} / \mathrm{HtrA} 2$ in the cytoplasmic and mitochondrial proteins using western blots. The release of Smac/DIABLO and Omi/ HtrA 2 into the cytoplasm was induced by Mcl-1 knockdown and inhibited by the overexpression of Mcl-1 (Fig. 1D).

Impact of Mcl-1 on cell cycle distribution in gastric cancer cells. To detect whether Mcl-1 could change cell cycle distribution, we performed flow cytometric analyses. Mcl-1 knockdown resulted in cell cycle arrest in the sub G1 phase of SNU638 and TMK1 cells. Cell cycle arrest in the sub-G1 phase induced by 5-FU treatment was inhibited by the overexpression of Mcl-1 (Fig. 2A). Next, we evaluated the effects of Mcl-1 on various cell cycle regulatory proteins in gastric cancer cells. As shown in Fig. 2B, the cyclin D1, P-cdc2, CDK4 and CDK6 protein levels were significantly decreased by Mcl-1 knockdown, and increased by the overexpression of Mcl-1 in SNU638 and TMK1 cells. However, p21 and p27 protein levels remained unchanged by knockdown and overexpression of Mcl-1.

Impact of Mcl-1 on the proliferation of gastric cancer cells. The proliferating cells, as determined by absorbance, were significantly increased in the pcDNA3.1-Mcl-1-transfected SNU638 cells compared to the empty-pcDNA3.1-transfected cells at 3 and 4 days ( $p=0.045$ and 0.049 , respectively), but there was no significant difference in the TMK1 cells. Moreover, in all tested cells, there was no significant difference in cell proliferation between Mcl-1 siRNA and scramble siRNA transfected cells (Fig. 3).

Impact of Mcl-1 on intracellular signaling pathways involved in the apoptosis and cell cycle distribution of gastric cancer cells. To explore the potential mechanisms involved in apoptosis and cell cycle distribution, we studied the effect of Mcl-1 on the stimulation of intracellular signaling pathways leading 


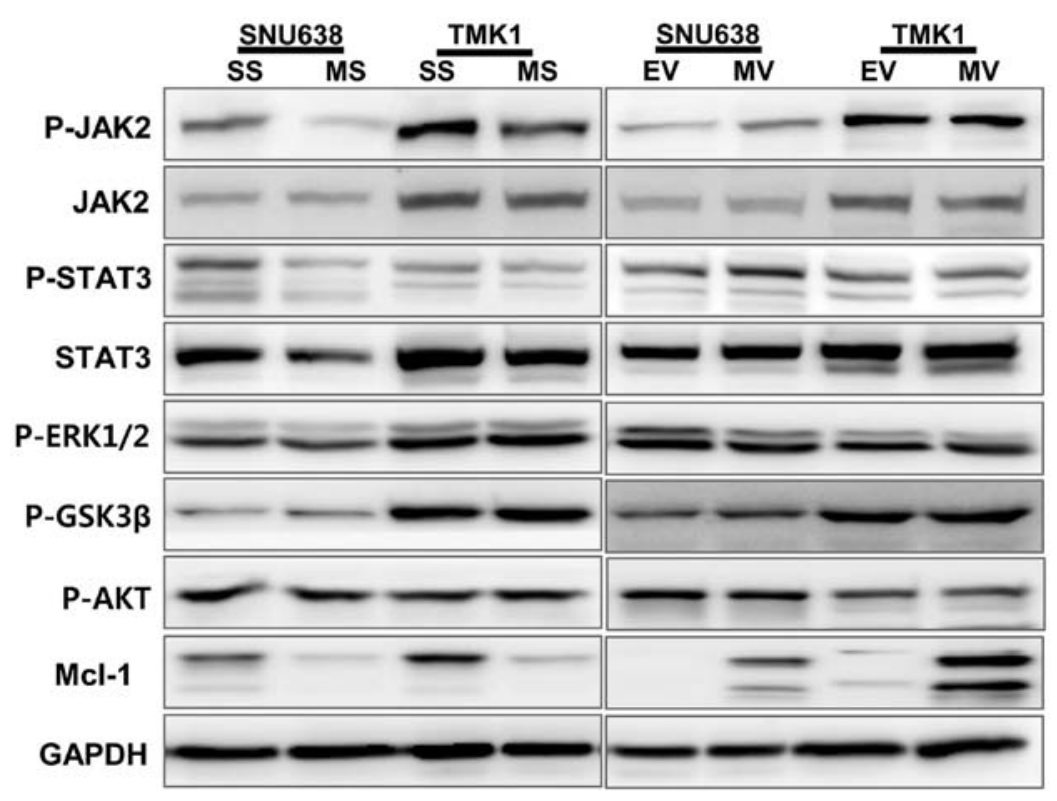

Figure 4. The impact of Mcl-1 on intracellular signaling pathways involved in the apoptosis and cell cycle distribution of gastric cancer cells. The phosphorylation levels of JAK2 and STAT3 were downregulated by Mcl-1 knockdown SNU638 and TMK1 cells and overexpression of Mcl-1 upregulated the phosphorylation of JAK2 and STAT3 in SNU638 cells. The phosphorylation levels of ERK1/2, GSK3 $\beta$ and AKT were not changed by knockdown or overexpression of Mcl-1. SS, scramble siRNA; MS, Mcl-1 siRNA; EV, empty-pcDNA3.1; MV, pcDNA3.1-Mcl-1; JAK2, Janus kinase 2; STAT3, signal transducers and activators of transcription-3; ERK1/2, extracellular signal-regulated kinase1/2; GSK3 $\beta$, glycogen synthase kinase-3 $\beta$.
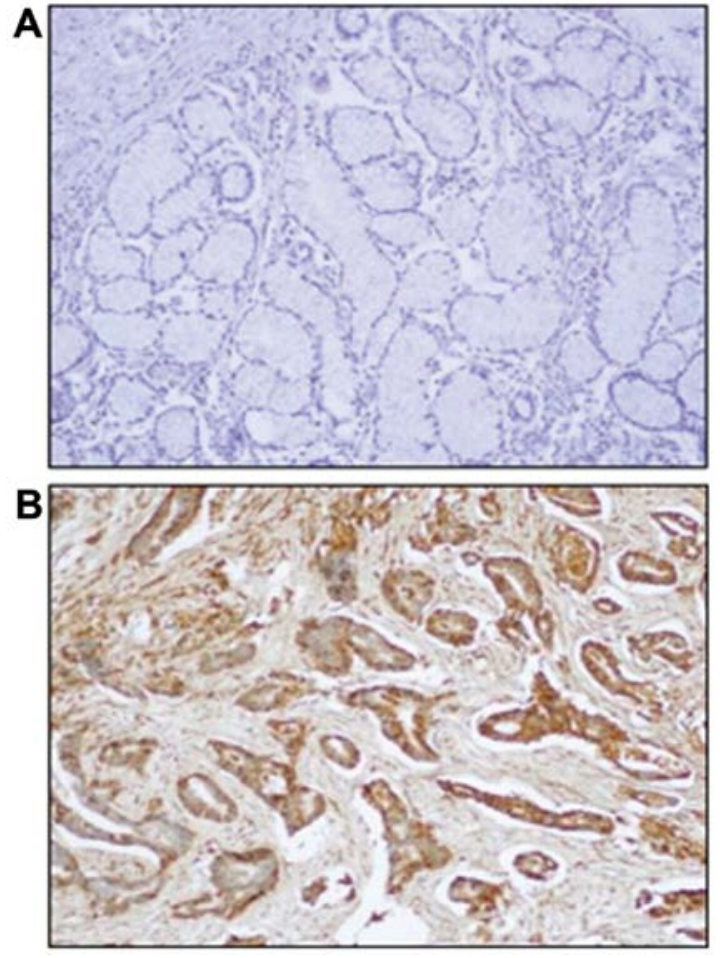

Figure 5. Expression of Mcl-1 protein in normal and gastric cancer tissues by immunohistochemistry. (A) The immunostaining of Mcl-1 protein did not or weakly stained the normal gastric mucosa. (B) The immunostaining of Mcl-1 protein was predominantly identified in the cytoplasm of cancer cells and not detectable in the tumor stroma. T, gastric cancer tissue; N, paired normal gastric mucosa.

to apoptosis and cell cycle distribution in SNU638 and TMK1 cells. The phosphorylation levels of JAK2 and STAT3 were downregulated by Mcl-1 knockdown SNU638 and TMK1 cells, and the overexpression of Mcl-1 upregulated the phosphorylation of JAK2 and STAT3 in SNU638 cells (Fig. 4). The phosphorylation levels of ERK1/2, GSK3 $\beta$ and AKT were not changed by knockdown and overexpression of Mcl-1.

Correlations between the expression of Mcl-1 and clinicopathological features in gastric cancers. To study the prognostic role of Mcl-1 in human gastric cancer progression, we investigated the expression of the Mcl-1 protein immunohistochemically in formalin-fixed, paraffin-embedded tissue blocks obtained from 139 gastric cancer patients with clinicopathological data. Survival and the correlation between immunostaining of Mcl-1 and clinicopathological parameters was analyzed. The immunostaining of Mcl-1 protein did not or weakly stained the normal gastric mucosa (Fig. 5A). The immunostaining of Mcl-1 protein was predominantly identified in the cytoplasm of cancer cells and was not detectable in the tumor stroma (Fig. 5A). The percentage of positive tumor cells and the staining intensity for each sample were recorded. For the 139 patient samples evaluated, positive Mcl-1 expression was observed in $41.0 \%$ of the gastric cancer tissues (57/139) (Table I). Immunostaining of Mcl-1 was significantly associated with age, tumor size, stage, depth of invasion and lymph node metastasis $(\mathrm{p}=0.001,0.013,0.003,0.022$ and 0.007 , respectively) (Table I). Moreover, overall survival for patients with positive Mcl-1 immunostaining was significantly shorter than for the negative patients $(\mathrm{p}=0.020)$ (Fig. 6).

Correlation between the expression of Mcl-1 and tumor cell proliferation or apoptosis in gastric cancers. The KI for the 139 tumors ranged from 14.8 to 86.5 , with a mean KI of $49.8 \pm 17.8$. The mean KI value of Mcl-1 positive tumors was $56.4 \pm 17.2$, and significantly lower than the KI of Mcl-1 negative tumors $(\mathrm{p}=0.018)$ (Table II). The AI for 139 tumors ranged from 0 to 
Table I. Correlation between Mcl-1 expression and the clinicopathological parameters of gastric cancer.

\begin{tabular}{|c|c|c|c|c|}
\hline \multirow[b]{2}{*}{ Characteristics } & \multirow[b]{2}{*}{$\begin{array}{c}\text { Total } \\
(\mathrm{n}=139)\end{array}$} & \multicolumn{2}{|c|}{ Mcl-1 } & \multirow[b]{2}{*}{ p-value } \\
\hline & & $\begin{array}{c}\text { Neg. } \\
(\mathrm{n}=82)\end{array}$ & $\begin{array}{l}\text { Pos. } \\
(\mathrm{n}=57)\end{array}$ & \\
\hline Age (years) & & & & 0.001 \\
\hline$<57$ & 57 & 43 & 14 & \\
\hline$\geq 57$ & 82 & 39 & 43 & \\
\hline Gender & & & & 0.366 \\
\hline Male & 94 & 53 & 41 & \\
\hline Female & 45 & 29 & 16 & \\
\hline Tumor size (cm) & & & & 0.013 \\
\hline$<4.0$ & 83 & 56 & 27 & \\
\hline$\geq 4.0$ & 56 & 26 & 30 & \\
\hline Stage & & & & 0.003 \\
\hline I & 66 & 46 & 20 & \\
\hline II & 19 & 14 & 5 & \\
\hline III & 34 & 16 & 18 & \\
\hline IV & 20 & 6 & 14 & \\
\hline Lauren classification & & & & 0.054 \\
\hline Intestinal & 84 & 44 & 40 & \\
\hline Diffuse & 54 & 38 & 16 & \\
\hline Mixed & 1 & 0 & 1 & \\
\hline Histologic type & & & & 0.190 \\
\hline Well differentiated & 39 & 19 & 20 & \\
\hline Moderately differentiated & 13 & 6 & 7 & \\
\hline Poorly differentiated & 81 & 54 & 27 & \\
\hline Signet ring cell & 6 & 3 & 3 & \\
\hline Depth of invasion (T) & & & & 0.022 \\
\hline $\mathrm{T} 1$ & 59 & 41 & 18 & \\
\hline $\mathrm{T} 2$ & 15 & 10 & 5 & \\
\hline T3 & 53 & 28 & 25 & \\
\hline $\mathrm{T} 4$ & 12 & 3 & 9 & \\
\hline Lymph node metastasis $(\mathrm{N})$ & & & & 0.007 \\
\hline No & 75 & 52 & 23 & \\
\hline N1-3 & 64 & 30 & 34 & \\
\hline Distant metastasis (M) & & & & NA \\
\hline M0 & 139 & 82 & 57 & \\
\hline M1 & 0 & 0 & 0 & \\
\hline
\end{tabular}

NA, not available; Neg, negative; Pos, positive.

6.3 with a mean $\mathrm{AI}$ of $1.8 \pm 1.4$. There was no significant difference between Mcl-1 expression and AI ( $\mathrm{p}=0.528)$ (Table II).

\section{Discussion}

Apoptosis is an essential mechanism for physiological and pathological cell death. The balance of cell growth and apoptosis determines normal tissue homeostasis. Inhibition of apoptosis plays a significant role in cancer promotion

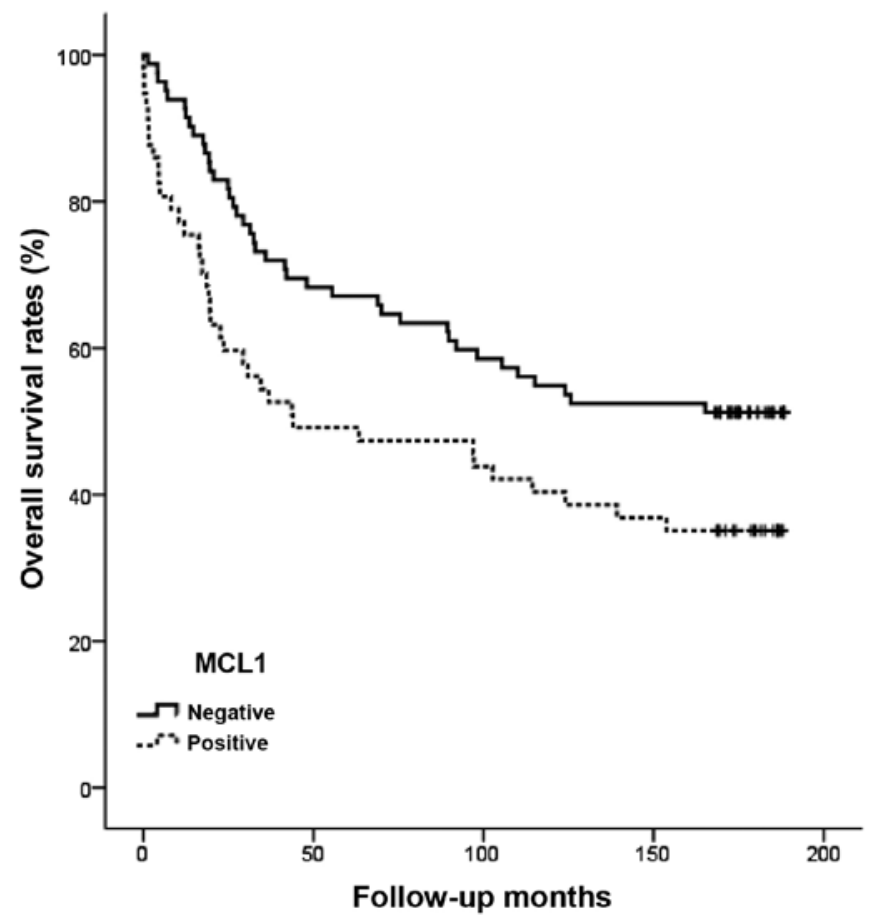

Figure 6. Kaplan-Meier survival curve correlating overall survival with positive expression (solid line) and negative expression (dotted line) of Mcl-1 $(\mathrm{p}=0.020)$.

Table II. Correlation between Mcl-1 expression and tumor cell proliferation or apoptosis in gastric cancers.

\begin{tabular}{lcccc}
\hline & & \multicolumn{2}{c}{ Mcl-1 expression } & \\
\cline { 3 - 4 } Indices & $\begin{array}{c}\text { Total } \\
(\mathrm{n}=139)\end{array}$ & $\begin{array}{c}\text { Neg. } \\
(\mathrm{n}=82)\end{array}$ & $\begin{array}{c}\text { Pos. } \\
(\mathrm{n}=57)\end{array}$ & p-value \\
\hline $\mathrm{KI}($ mean $\pm \mathrm{SD})$ & $49.8 \pm 17.8$ & $38.8 \pm 13.0$ & $56.4 \pm 17.2$ & 0.018 \\
$\mathrm{AI}($ mean \pm SD) & $1.8 \pm 1.4$ & $1.7 \pm 1.7$ & $1.9 \pm 1.4$ & 0.528
\end{tabular}

KI, Ki-67 labelling index; AI, apoptotic index; SD, standard deviation; Neg, negative; Pos, positive.

and resistance to chemotherapy $(6,7)$. Several inhibitors of apoptosis proteins (IAPs) have been identified, including the Bcl-2, p53 and p73 families (8-11). Mcl-1 has been identified as a member of anti-apoptotic Bcl-2 family proteins and is frequently expressed in various cancer tissues (12-14). Our study showed that Mcl-1 knockdown induced apoptosis, while the overexpression of Mcl-1 inhibited apoptosis in gastric cancer cells, suggesting a role of Mcl-1 in the alteration of the invasive and oncogenic phenotypes of gastric cancer cells.

Generally, there are two main pathways in apoptosis: the mitochondrial pathway, which involves the release of cytochrome $\mathrm{C}$ from the mitochondria into the cytoplasm, and the cell surface pathway, which is stimulated by cell surface death receptors such as Fas and TNFR. Both pathways share the activation of caspases, which are considered to be crucial effectors of the cell death machinery $(6,7)$. In our study, the 
cleaved caspase- $3,-7$, and PARP expression levels were upregulated in gastric cancer cells after Mcl-1 knockdown and downregulated after the overexpression of Mcl-1.

Mcl-1 inhibits the progression of apoptosis by interacting with pro-apoptotic proteins such as Bak and Bax on the mitochondrial membrane preventing such molecules from dimerizing to form pro-apoptotic pores, and the subsequent release of cytochrome C into the cytoplasm (12-14). Recently, it has been found that Smac/DIABLO and Omi/HtrA2 bind IAPs and promote apoptosis. The apoptotic activity of Smac/ DIABLO and Omi/HtrA2 is regulated by the release of Smac/ DIABLO and Omi/HtrA2 from the mitochondrial membrane to the cytoplasm, following apoptotic stimuli (27-29). In our study, the release of Smac/DIABLO and Omi/HtrA2 into the cytoplasm was induced by Mcl-1 knockdown and inhibited by the overexpression of Mcl-1. Therefore, the anti-apoptotic mechanism of Mcl-1 is mediated by the direct inhibition of caspase activity and is negatively regulated by the endogenous IAPs antagonist Smac/DIABLO and Omi/HtrA2 in gastric cancer cells.

The cell cycle is the cascade of events that promote a growing cell to duplicate all its components and ultimately split into two daughter cells. The cell cycle is governed by CDK, and the activity is regulated by the positive regulators, including the cyclins and CDK binding proteins, by negative regulators, including the CDKIs, and by phosphorylation and dephosphorylation events $(30,31)$. In addition, Cdc2 is one of the most important regulated kinases of cell cycle. Its activity is regulated positively by cyclin B1 and negatively by CKI (32). Our study showed that knockdown of Mcl-1 induced cell cycle arrest by decreasing cyclin D1, cdc2, and CDK 4 and 6 in gastric cancer cells. In contrast, overexpression of Mcl-1 inhibited cell cycle arrest. However, CDKIs, such as p21 and p27, remained unchanged by the knockdown and overexpression of Mcl-1. Alterations in genes involved in the regulation of cell cycle progression are frequent events in human cancers (30-32). Therefore, Mcl-1 may contribute to gastric cancer progression via cell cycle regulation by controlling cyclin, cdc2 and CDK expression.

Cell proliferation and apoptosis is a tightly regulated process under the control of multiple intracellular signaling pathways including AKT, ERK1/2, GSK $3 \beta$, JAK2 and STAT3 (33-36). In our study, the phosphorylation levels of JAK2 and STAT3 were significantly blocked by the knockdown of Mcl-1. Also, the phosphorylation levels of JAK2 and STAT3 were increased by overexpression of Mcl-1. The results suggest that Mcl-1 might mainly regulate gastric cancer cell growth through the JAK2 and STAT3 signaling pathways.

Previously, expression of Mcl-1 has been reported to be highly expressed in various human cancer types and has also been associated with tumor progression and adverse clinical outcomes (15-21). In our study, Mcl-1 expression was significantly increased in gastric cancer tissues compared to normal gastric mucosa tissues, and associated with age, tumor size, stage, depth of invasion, lymph node metastasis and poor survival. This result is in agreement with the results of previous studies (18-20). Previously, silencing the Mcl-1 gene using antisense oligonulceotides produced a significant increase in apoptosis and decrease in cell growth, and in combination with chemotherapy displayed synergistic antitumor activity in gastric cancer cells $(37,38)$. Therefore, Mcl-1 may be considered as a potential therapeutic target in the treatment of colorectal cancer.

Tumor volume is balanced by tumor cell proliferation and apoptosis, and while both activities often increase in tandem, proliferative activity is generally linked to tumor progression (3-7). Therefore, we evaluated the correlation between Mcl-1 expression and tumor cell proliferation or apoptosis in gastric cancer tissues. In our study, the mean KI value of Mcl-1-positive tumors was significantly lower than that of Mcl-1-negative tumors. However, there was no significant difference between Mcl-1 expression and AI.

Taken together, we found that Mcl-1 plays an important role in human gastric cancer progression by modulating tumor cell proliferation and apoptosis and may be used as a molecular marker for the prediction of clinical outcomes in gastric cancer.

\section{Acknowledgements}

This work was supported by research funds from the Research Institute of Clinical Medicine, Chonnam National University Hospital in 2013 (CRI 13025-1), Republic of Korea.

\section{References}

1. Guggenheim DE and Shah MA: Gastric cancer epidemiology and risk factors. J Surg Oncol 107: 230-236, 2013.

2. Krejs GJ: Gastric cancer: Epidemiology and risk factors. Dig Dis 28: 600-603, 2010

3. Allan AL, Vantyghem SA, Tuck AB and Chambers AF: Tumor dormancy and cancer stem cells: Implications for the biology and treatment of breast cancer metastasis. Breast Dis 26: 87-98, 2006-2007.

4. Brábek J, Mierke CT, Rösel D, Veselý P and Fabry B: The role of the tissue microenvironment in the regulation of cancer cell motility and invasion. Cell Commun Signal 8: 22, 2010.

5. Chaffer CL and Weinberg RA: A perspective on cancer cell metastasis. Science 331: 1559-1564, 2011.

6. Kiechle FL and Zhang X: Apoptosis: biochemical aspects and clinical implications. Clin Chim Acta 326: 27-45, 2002.

7. Schultz DR and Harrington WJ Jr: Apoptosis: Programmed cell death at a molecular level. Semin Arthritis Rheum 32: 345-369, 2003.

8. Ola MS, Nawaz $\mathrm{M}$ and Ahsan $\mathrm{H}$ : Role of Bcl-2 family proteins and caspases in the regulation of apoptosis. Mol Cell Biochem 351: 41-58, 2011.

9. Llambi F and Green DR: Apoptosis and oncogenesis: Give and take in the BCL-2 family. Curr Opin Genet Dev 21: 12-20, 2011.

10. Weyhenmeyer B, Murphy AC, Prehn JH and Murphy BM: Targeting the anti-apoptotic Bcl-2 family members for the treatment of cancer. Exp Oncol 34: 192-199, 2012.

11. Davids MS and Letai A: Targeting the B-cell lymphoma/ leukemia 2 family in cancer. J Clin Oncol 30: 3127-3135, 2012.

12. Thomas LW, Lam C and Edwards SW: Mcl-1; the molecular regulation of protein function. FEBS Lett 584: 2981-2989, 2010.

13. Akgul C: Mcl-1 is a potential therapeutic target in multiple types of cancer. Cell Mol Life Sci 66: 1326-1336, 2009.

14. Quinn BA, Dash R, Azab B, et al: Targeting Mcl-1 for the therapy of cancer. Expert Opin Investig Drugs 20: 1397-1411, 2011.

15. Zhang T, Zhao C, Luo L, Zhao H, Cheng J and Xu F: The expression of Mcl-1 in human cervical cancer and its clinical significance. Med Oncol 29: 1985-1991, 2012.

16. Luo L, Zhang T, Liu H, Lv T, Yuan D, Yao Y, Lv Y and Song Y: MiR-101 and Mcl-1 in non-small-cell lung cancer: Expression profile and clinical significance. Med Oncol 29: 1681-1686, 2012.

17. Henderson-Jackson EB, Helm J, Ghayouri M, Hakam A, Nasir A, Leon M, Bui M, Yeatman T and Coppola D: Correlation between Mcl-1 and pAKT protein expression in colorectal cancer. Int $\mathrm{J}$ Clin Exp Pathol 3: 768-774, 2010. 
18. Likui W, Qun L, Wanqing Z, Haifeng S, Fangqiu L and Xiaojun L: Prognostic role of myeloid cell leukemia-1 protein (Mcl-1) expression in human gastric cancer. J Surg Oncol 100: 396-400, 2009.

19. Maeta Y, Tsujitani S, Matsumoto S, et al: Expression of Mcl-1 and $\mathrm{p} 53$ proteins predicts the survival of patients with $\mathrm{T} 3$ gastric carcinoma. Gastric Cancer 7: 78-84, 2004.

20. Tsujitani S, Saito H, Wakatsuki T, Ikeguchi M, Shirabe K, Morita M, Kakeji Y, Yano T and Maehara Y: Relationship between expression of apoptosis-related proteins and the efficacy of postoperative chemotherapy in patients with $\mathrm{T} 3$ gastric cancer. Surg Today 42: 225-232, 2012.

21. Akagi H, Higuchi H, Sumimoto H, et al: Suppression of myeloid cell leukemia-1 (Mcl-1) enhances chemotherapy-associated apoptosis in gastric cancer cells. Gastric Cancer 16: 100-110, 2013.

22. Mandelin AM II and Pope RM: Myeloid cell leukemia-1 as a therapeutic target. Expert Opin Ther Targets 11: 363-373, 2007.

23. Perciavalle RM and Opferman JT: Delving deeper: MCL-1's contributions to normal and cancer biology. Trends Cell Biol 23: 22-29, 2013.

24. Hartman ML and Czyz M: Anti-apoptotic proteins on guard of melanoma cell survival. Cancer Lett 331: 24-34, 2013.

25. Bose P and Grant S: Mcl-1 as a therapeutic target in acute myelogenous leukemia (AML). Leuk Res Rep 2: 12-14, 2013.

26. Frederick L, Greene D and Irvin D: AJCC cancer staging manual. 6th edition. Springer-Verlag, New York, pp17-57, 2002.

27. Obexer $\mathrm{P}$ and Ausserlechner MJ: X-linked inhibitor of apoptosis protein - a critical death resistance regulator and therapeutic target for personalized cancer therapy. Front Oncol 4: 197, 2014.

28. de Almagro MC and Vucic D: The inhibitor of apoptosis (IAP) proteins are critical regulators of signaling pathways and targets for anti-cancer therapy. Exp Oncol 34: 200-211, 2012.
29. Kilbride SM and Prehn JH: Central roles of apoptotic proteins in mitochondrial function. Oncogene 32: 2703-2711, 2013.

30. Graña X and Reddy EP: Cell cycle control in mammalian cells: Role of cyclins, cyclin dependent kinases (CDKs), growth suppressor genes and cyclin-dependent kinase inhibitors (CKIs). Oncogene 11: 211-219, 1995.

31. Soták M, Sumová A and Pácha J: Cross-talk between the circadian clock and the cell cycle in cancer. Ann Med 46: 221-232, 2014.

32. FisherD, KrasinskaL, Coudreuse D and Novák B: Phosphorylation network dynamics in the control of cell cycle transitions. J Cell Sci 125: 4703-4711, 2012.

33. Yu HG, Ai YW, Yu LL, et al: Phosphoinositide 3-kinase/Akt pathway plays an important role in chemoresistance of gastric cancer cells against etoposide and doxorubicin induced cell death. Int J Cancer 122: 433-443, 2008.

34. Choi IJ, Kim JS, Kim JM, Jung HC and Song IS: Effect of inhibition of extracellular signal-regulated kinase 1 and 2 pathway on apoptosis and bcl-2 expression in Helicobacter pylori-infected AGS cells. Infect Immun 71: 830-837, 2003.

35. McCubrey JA, Steelman LS, Bertrand FE, et al: GSK-3 as potential target for therapeutic intervention in cancer. Oncotarget 5: 2881-2911, 2014.

36. Amoyel M, Anderson AM and Bach EA: JAK/STAT pathway dysregulation in tumors: A Drosophila perspective. Semin Cell Dev Biol 28: 96-103, 2014.

37. Wacheck V, Cejka D, Sieghart W, Losert D, Strommer S, Crevenna R, Monia BP and Selzer E: Mcl-1 is a relevant molecular target for antisense oligonucleotide strategies in gastric cancer cells. Cancer Biol Ther 5: 1348-1354, 2006.

38. Zangemeister-Wittke U and Huwiler A: Antisense targeting of Mcl-1 has therapeutic potential in gastric cancer. Cancer Biol Ther 5: 1355-1356, 2006. 